ESAIM: PROCEEDINGS, October 2007, Vol.20, 29-43

Mohammed-Najib Benbourhim, Patrick Chenin, Abdelhak Hassouni \& Jean-Baptiste Hiriart-Urruty, Editors

\title{
APPROXIMATION BY NEW FAMILIES OF UNIVARIATE SYMMETRICAL B-SPLINES
}

\author{
El Bachir AMEUR ${ }^{1}$, Hamid MRAOUI ${ }^{2}$ AND Driss SBIBIH ${ }^{1}$
}

\begin{abstract}
In this paper we prove that there exists a unique positive symmetrical univariate B-spline with minimal support. It is obtained as linear combination of a minimal number of successive classical B-splines with multiple knots in the space, $\mathcal{S}_{d}^{r}$, of cardinal polynomial splines of class $\mathcal{C}^{r}$ and degree $d$. Next, we show that the approximation order in the space generated by the integer translates of this B-spline is not optimal. However it can be used for geometrical design where the small support is appreciated but the approximation order is not crucial. To have a higher approximation order, we define the B-splines of high order by recurrence and by convolution with the characteristic function of the interval $[0,1]$. We use these B-splines to study the cardinal interpolation and we show that it is correct in the sense of Schoenberg. Finally, we give the explicit expression of interplant operators associated with some of these B-splines.
\end{abstract}

Résumé. Dans cet article, nous montrons l'existence et l'unicité d'une spline symétrique à support minimal qui s'écrit comme combinaison linéaire d'un nombre minimal de B-spline successives de l'espace $\mathcal{S}_{d}^{r}$ des splines polynomiales cardinales de degré $d$ et de régularité $r$. Nous démontrons que l'ordre d'approximation dans l'espace engendré par les translatés entières de cette B-spline n'est pas optimal. Cependant, leur utilisation dans le dessin géométrique, où l'ordre d'approximation n'est pas crucial mais où un support de longueur réduit est recommondé, pourrait être très utile. Pour avoir un ordre d'approximation élevé, nous définissons par récurrence de nouvelles familles de B-splines cardinales symétriques. Ensuite, nous étudions l'unisolvance du problème d'interpolation basé sur ces B-splines. Nous donnons enfin, des exemples de calcul des coefficients des splines fondamentales associées à quelques éléments de degrés faibles de ces nouvelles familles.

\section{INTRODUCTION}

It is well known that the classical cardinal B-spline of order $d+1$ and degree $d$ with simple knots $0,1, \ldots, d+1$ is of class $\mathcal{C}^{d-1}$ and of support [0,d+1]. Moreover, it is symmetric and satisfies other properties (see e.g. [4,5,15]). The degree, class and length of support of this B-spline depend all on the integer $d$. However, a high regularity leads to a B-spline with a large support. Consequently, the approximation in the space spanned by its integer translates requires the resolution of a linear system with a very large size, and a full associated matrix. Therefore the linear system is difficult to solve. So, one way to construct B-splines of high regularity and small support is to use the classical cardinal B-splines with multiple knots. In this case, (see e.g. $[4,6,15]$ ), one can obtain a finite family of B-splines which form a partition of unity, but in general they are not symmetric. Our aim is to define new polynomial symmetrical B-splines, associated with the uniform knot sequence $\tau=\mathbb{Z}$, with small

\footnotetext{
1 Département d'Informatique, Faculté des Sciences et Techniques, Errachidia, Maroc;e-mail: eb_ameur@yahoo.fr

2 Université Mohammed I, Ecole Supérieure de Technologie, Laboratoire MATSI, Oujda, Maroc;

e-mail: sbibih@yahoo.fr \& e-mail: hamid_mraoui@yahoo.fr
} 
supports, which depend only on $d$, but their class and degree depend on $d$ and another nonnegative integer $r$. This idea is generalized to the bivariate case, (see [9-11]), the authors construct B-splines of class $\mathcal{C}^{r}$ and small supports on three and four directional meshes of the plane.

Let $\varphi$ be one of these B-splines and $\widehat{\varphi}$ its Fourier transform. We denote by $S(\varphi)$ the space generated by the integer translates of $\varphi$, and for $h>0, S_{h}(\varphi)=\left\{\sigma_{h} f, f \in S(\varphi)\right\}$, where $\left(\sigma_{h} f\right)(x)=f\left(\frac{x}{h}\right)$.

In this paper, we study cardinal interpolation problem with new families of univariate cardinal B-splines of high regularity and small support which are symmetric and form a partition of unity. A cardinal interpolant to a sequence $f$ on $\mathbb{Z}$ is a spline function in $S(\varphi)$ of the form $\sum_{i \in \mathbb{Z}} a_{i}(\varphi) \varphi(\cdot-i)$ and which matches $f$ on $\mathbb{Z}$, i.e.,

$$
\sum_{i \in \mathbb{Z}} a_{i}(\varphi) \varphi(j-i)=f(j), \text { for all } j \in \mathbb{Z}
$$

where $f$ is a sufficiently smooth function.

As in [14], we say that the cardinal interpolation with $\varphi$ is correct if for any bounded function $f$ there exists a unique bounded solution $a=\left\{a_{i}(\varphi), i \in \mathbb{Z}\right\}$ of equation (1).

The solution $L=\sum_{i \in \mathbb{Z}} c_{i}(\varphi) \varphi(\cdot-i)$ of $(1)$ which interpolates the data $\left\{\delta_{0, j}\right\}$, where $\delta_{0, j}$ is the Kronecker symbol, is called cardinal fundamental spline function associated with $\varphi$. The cardinal interpolant to a sequence $f$ on $\mathbb{Z}$ given by (1) can be written in Lagrange form

$$
(\mathcal{I} f)(x)=\sum_{j \in \mathbb{Z}} f(j) L(x-j)
$$

Therefore, solving equation (1) is equivalent to determine the cardinal fundamental spline $L$. On the other hand, if we denote $\widetilde{\varphi}(w)=\sum_{j \in \mathbb{Z}} \varphi(j) e^{-\imath w j}$ (resp. $\widetilde{a}(w)=\sum_{j \in \mathbb{Z}} a_{j}(\varphi) e^{-\imath w j}$ ) the symbol of the function $\varphi$ (resp. the sequence $a$ ), then, according to [14], the cardinal interpolation with $\varphi$ is correct if and only if the symbol $\tilde{\varphi}$ of $\varphi$ does not vanish. In this case, the cardinal fundamental spline $L$ has an exponential decay and the symbol of the sequence $\left\{c_{i}(\varphi)\right\}$ is given by $\widetilde{c}(w)=\frac{1}{\tilde{\varphi}(w)}$. Moreover, for $h>0$, the optimal approximation order of the interpolant $\mathcal{I}_{h}=\sigma_{h} \mathcal{I} \sigma_{\frac{1}{h}}$ in $S_{h}(\varphi)$ coincides with the following so-called Fix-Strang condition of order $n$ of $\varphi$ (see $[16])$

$$
\left\{\begin{array}{cc}
\widehat{\varphi}(0) & =1, \\
D^{k} \widehat{\varphi}(2 \pi j) & =0,
\end{array} \text { for } j \in \mathbb{Z} \backslash\{0\} \text { and } k=0, \ldots, n .\right.
$$

The paper is organized as follows. In Section 2, we give some preliminary results about the classical cardinal B-splines with multiple knots in the space of polynomial spline functions $\mathcal{S}_{d}^{r}$ of class $\mathcal{C}^{r}$ and degree $d$ associated with the uniform knot sequence $\mathbb{Z}$. In Section 3, we prove the existence and the uniqueness of a symmetrical B-spline with minimal support. This B-spline, noted $M$ and obtained as a linear combination of a minimal number of successive classical cardinal B-splines with multiple knots, is positive and its integer translates form a partition of unity, i.e., $\sum_{j \in \mathbb{Z}} M(\cdot-j)=1$. We show that approximation order in the space $S(M)$ is not optimal, so these splines can be useful for the geometrical design where the order of convergence is not crucial but small supports are recommended. In Section 4, we construct, by recurrence and convolution with the the characteristic function of [0,1], a new family of B-splines with higher approximation orders and based on $M$. Next, we study the cardinal interpolation problem in the space $S(\varphi)$ when $\varphi$ is one of these later B-splines. We prove that the symbol of $\varphi$ is positive and therefore the cardinal interpolation with $\varphi$ is correct. Finally, as examples, we give the explicit expressions of fundamental splines associated with some of these B-splines.

\section{Preliminary Results}

Let $r, d$ be two positive integers such that $r<d$. We denote by $\mathcal{S}_{d}^{r}$ the space of cardinal polynomial splines of class $C^{r}$ and degree $d$, with the set $\mathbb{Z}$ of all integers as the knot sequence. Let $\mu=d-r$ be the multiplicity 
of each knot. We assume that $d \geq 1$ and $0 \leq r \leq d-1$, then $1 \leq \mu \leq d-1$. In the following, we put $e=\left\lfloor\frac{d+1}{\mu}\right\rfloor$ and $\rho=d+1-\mu e$, where $\lfloor x\rfloor=\max \{n \in \mathbb{Z}: n \leq x\}$.

In order to define the B-splines basis for the space $\mathcal{S}_{d}^{r}$, we consider the following knot sequence

$$
\cdots, x_{-1}=-1, x_{0}=0, \cdots, x_{\mu-1}=0, x_{\mu}=1, \cdots, x_{2 \mu-1}=1, x_{2 \mu}=2, \cdots
$$

Let $n g_{i}=\#\left\{j \in \mathbb{Z}: j \leq i\right.$ and $\left.x_{j}=x_{i}\right\}$ and $n d_{i}=\#\left\{j \in \mathbb{Z}: j \leq i+d+1\right.$ and $\left.x_{j}=x_{i+d+1}\right\}$. Then we have the following result.

Theorem 1.1. There exists a unique B-splines basis $\left(N_{i}\right)_{i \in \mathbb{Z}}$ for the space $\mathcal{S}_{d}^{r}$ which satisfies the following properties

(i) $\operatorname{supp} N_{i}=\left[x_{i}, x_{i+d+1}\right]$,

(ii) $N_{i}$ is positive on $] x_{i}, x_{i+d+1}[$,

(iii) $N_{i}$ is of class $C^{r-1+n g_{i}}$ at $x_{i}$ and of class $C^{r-1+n d_{i}}$ at $x_{i+d+1}$,

(iv) $\sum_{i \in \mathbb{Z}} N_{i}=1$.

Proof. It derives from the classical properties of B-splines, see [15].

From the above properties, we deduce that for each integer $i$, there are $\mu-\rho$ B-splines $N_{i \mu+j}, j=0, \cdots, \mu-$ $\rho-1$, which have as support $[i, i+e]$ and $\rho$ B-splines $N_{i \mu+j}, j=\mu-\rho, \cdots, \mu-1$, with support $[i, i+e+1]$. Moreover, these B-splines are all of class $C^{r+j}$ at $i$, but the $\mu-\rho$ first ones are of class $C^{d-\rho-1-j}$ at $i+e$ and the $\rho$ later ones are of class $C^{d+\mu-\rho-1-j}$ at $i+e+1$.

It is well known that when $\mu=1$ (simple knots), the B-splines $N_{i}$ are symmetrical w.r.t. the middle of their supports and they can be easily obtained by translation, i.e., $N_{i}(x)=N_{0}(x-i)$. When $\mu>1$, there are also some specific properties of symmetry and periodicity.

Proposition 1.2. For $\mu \geq 1$, the B-splines $N_{i}$ satisfy

(i) $N_{i \mu+j}(x)=N_{j}(x-i)$, for all $x \in \mathbb{R}, i \in \mathbb{Z}$ and $0 \leq j \leq \mu-1$;

(ii) $N_{j}(x)=N_{\mu-\rho-j-1}(e-x), 0 \leq j \leq\left\lfloor\frac{\mu-\rho-1}{2}\right\rfloor$;

(iii) $N_{j}(x)=N_{2 \mu-\rho-j-1}(e+1-x), \mu-\rho \leq j \leq \mu-\rho+\left\lfloor\frac{\rho-1}{2}\right\rfloor$.

Proof. ( $i$ ) derives from the fact that the knots are uniform and of multiplicity $\mu$. (ii) and (iii) can be easily proved by using Theorem 2.1 or the recurrence relation of B-splines.

\section{Symmetrical B-SPlines With Minimal SUPPORT}

In this section we show that there exists a unique symmetrical B-spline with minimal support in $\mathcal{S}_{d}^{r}$. It is obtained as linear combination of a minimal number of successive B-splines satisfying interesting properties. To establish the main result, we need the following lemma.

Lemma 2.1. Let $L$ be a spline function in $S_{d}^{r}$ of support $[0, m], m \in \mathbb{N}^{*}$. If $L$ is a linear combination of successive $l$ B-splines $N_{i}$, then $\sum_{i \in \mathbb{Z}} L(\cdot-i)=1$ implies that $l \geq \mu$.

Proof. As $L$ does not vanish on ]0,1[, and it is linear combination of successive $l$ B-splines $N_{i}$, it can be written in the form

$$
L=\sum_{i=k}^{l+k-1} a_{i} N_{i}, \text { where } k \in\{0, \ldots, \mu-1\} \text { and } a_{i} \in \mathbb{R}^{*} .
$$

Then, for $l=n * \mu+\theta$, with $0 \leq \theta \leq \mu-1$, we obtain

$$
L=\sum_{i=0}^{n-1} \sum_{j=0}^{\mu-1} a_{i \mu+k+j} N_{i \mu+k+j}+\sum_{j=0}^{\theta-1} a_{n \mu+k+j} N_{n \mu+k+j}=\sum_{i=0}^{n} \sum_{j=0}^{\mu-1} a_{i \mu+k+j} N_{i \mu+k+j},
$$


with $a_{n \mu+k+j}=0$ for $\theta \leq j \leq \mu-1$.

Consequently, we have

$$
\sum_{i \in \mathbb{Z}} L(\cdot-i)=\sum_{i \in \mathbb{Z}} \sum_{s=0}^{n} \sum_{j=0}^{\mu-1} a_{s \mu+k+j} N_{s \mu+k+j}(\cdot-i) N_{(s+i) \mu+k+j}=\sum_{i \in \mathbb{Z}} \sum_{j=0}^{\mu-1}\left(\sum_{s=0}^{n} a_{s \mu+k+j}\right) N_{i \mu+k+j}
$$

Since

$$
\sum_{i \in \mathbb{Z}} L(\cdot-i)=1=\sum_{i \in \mathbb{Z}} N_{i}=\sum_{i \in \mathbb{Z}} \sum_{j=0}^{\mu-1} N_{i \mu+k+j}=\sum_{i \in \mathbb{Z}} \sum_{j=0}^{\mu-1}\left(\sum_{s=0}^{n} a_{s \mu+k+j}\right) N_{i \mu+k+j},
$$

we deduce that $\sum_{s=0}^{n} a_{s \mu+k+j}=1$, for all $0 \leq j \leq \mu-1$. Thus, for each $j \in\{0, \ldots, \mu-1\}$, there exists $s \in\{0, \ldots, n\}$ such that $a_{s \mu+k+j} \neq 0$, and therefore $l \geq \mu$.

Theorem 2.2. In $S_{d}^{r}$ there exists a unique symmetrical B-spline $M$ with minimal support $\left[0, m_{0}\right]$, such that

i) $M$ is symmetric.

ii) $M(x)>0$ for $0<x<m_{0}$.

iii) $M$ is a linear combination of a minimal number of successive B-splines $N_{i}$.

iv) $\sum_{i \in \mathbb{Z}} M(\cdot-i)=1$

The length of the support of $M$ is equal to $e$ if $\rho=0$ and equal to $e+1$ if $\rho>0$.

Proof. Let us denote $S=\left\{\sum_{i=k}^{l+k-1} a_{i} N_{i}, l \in \mathbb{N}^{*}, k \in\{0, \ldots, \mu-1\}\right.$ and $\left.a_{i} \in \mathbb{R}^{*}\right\}$. Let $M=\sum_{i=k}^{l+k-1} a_{i} N_{i} \in S$ such that $\sum_{i \in \mathbb{Z}} M(\cdot-i)=1$. According to Lemma 2.1, we have $l \geq \mu$. Moreover, $M$ can be written in the form

$$
M=\sum_{s=0}^{n} \sum_{j=0}^{\mu-1} a_{s \mu+k+j} N_{s \mu+k+j}, \text { where } n \text { is such that } l=n * \mu+\theta, 0 \leq \theta \leq \mu-1 .
$$

Assume that $l=\mu$, i.e., $n=1$ and $\theta=0$. According to Lemma 2.1, we have $\sum_{s=0}^{1} a_{s \mu+k+j}=1$, where $a_{\mu+k+j}=0$ for $0 \leq j \leq \mu-1$. Consequently, $a_{k+j}=1$ for all $0 \leq j \leq \mu-1$ and $M$ can be written as

$$
M=\sum_{j=0}^{\mu-1} N_{k+j}=\sum_{j=k}^{k+\mu-1} N_{j}
$$

For $\rho=0$, the support of all B-splines $N_{i}$ are of length $e$, then in this case the unique minimal supported spline of type (2) is $M=\sum_{j=0}^{\mu-1} N_{j}$.

By construction, $M$ satisfies the properties ii), iii), and iv). To show that $M$ is symmetric, it suffices to remark that $M$ can be written as

$$
M= \begin{cases}\sum_{j=0}^{\frac{\mu}{2}-1}\left(N_{j}+N_{\mu-1-j}\right) & \text { if } \mu \text { is even } \\ \sum_{j=0}^{\frac{\mu-1}{2}-1}\left(N_{j}+N_{\mu-1-j}\right)+N_{\frac{\mu-1}{2}} & \text { if } \mu \text { is odd }\end{cases}
$$

Since $N_{j}+N_{\mu-1-j}$ and $N_{\frac{\mu-1}{2}}$ are symmetric with respect to $\frac{e}{2}$, we deduce that $M$ is symmetric. 
When $\rho>0$, we have $\mu-\rho$ successive B-splines $N_{i}$ of support $[0, e]$ and $\rho$ B-splines of support $[0, e+1]$. Assume that $k \leq \mu-\rho$. In this case, a spline $M=\sum_{j=k}^{k+\mu-1} N_{j}=\sum_{j=k}^{\mu-1} N_{j}+\sum_{j=0}^{k-1} N_{\mu+j}$ is of support [0,e+1]. Moreover, it is easy to see that $M$ is of class $C^{(r-1)+k+1}$ at 0 and of class $C^{(r-1)+\mu-\rho-k+1}$ at $e+1$. Consequently, so that $M$ is symmetrical on $[0, e+1]$, we must have $k+1=\mu-\rho-k+1$, i.e., $\mu-\rho=2 k$. Hence, if $\mu-\rho$ is even, there exists a unique spline $M=\sum_{j=\frac{\mu-\rho}{2}}^{\frac{\mu-\rho}{2}+\mu-1} N_{j}$ of minimal support $[0, e+1]$ which satisfies the properties ii), iii) and iv).

To prove that $M$ is symmetric, it suffices to remark that it can be written as

$$
M= \begin{cases}\sum_{j=0}^{k-1}\left(N_{k+j}+N_{\mu+k-(1+j)}\right)+\sum_{j=\mu-\rho}^{\mu-\varrho-1}\left(N_{j}+N_{\mu-1-(j-\mu+\rho)}\right) & \text { if } \rho=2 \varrho . \\ \sum_{j=0}^{k-1}\left(N_{k+j}+N_{\mu+k-(1+j)}\right)+\sum_{j=\mu-\rho}^{(\mu-\rho)+\varrho-1}\left(N_{j}+N_{\mu-1-(j-\mu+\rho)}\right)+N_{\mu-\rho+\varrho} & \text { if } \rho=2 \varrho+1 .\end{cases}
$$

As $N_{k+j}+N_{\mu+k-(j+1)},\left(N_{j}+N_{\mu-1-(j-\mu+\rho)}\right)$ and $N_{\mu-\rho+\frac{\rho-1}{2}}$ are symmetric with respect to $\frac{e+1}{2}$, we deduce that $M$ is symmetric.

In the case where $\mu-\rho$ is odd, there exists no spline in $S$ which is symmetric, of support ]0,e+1[ and linear combination of $\mu$ successive B-splines $N_{i}$. Consequently, it is necessary to take $l=\mu+1$.

Assume that $l=\mu+1$, i.e., $n=1$ and $\theta=1$. In this case, $M$ can be written as

$$
M=\sum_{s=0}^{1} \sum_{j=0}^{\mu-1} a_{s \mu+k+j} N_{s \mu+k+j}
$$

Since $\sum_{s=0}^{1} a_{s \mu+k+j}=1$, for all $0 \leq j \leq \mu-1$, where $a_{\mu+k+j}=0,1 \leq j \leq \mu-1$, we have $a_{k}+a_{\mu+k}=1$ and $a_{k+j}=1$, for all $1 \leq j \leq \mu-1$. Thus, from (3) we get

$$
M=a_{k} N_{k}+\sum_{i=k+1}^{k+\mu-1} N_{i}+a_{k+\mu} N_{k+\mu}
$$

Assume that $k<\mu-\rho$. In this case, $M$ is of support $[0, e+1]$ and it is easy to see that $M$ is of class $C^{(r-1)+k+1}$ at 0 and of class $C^{(r-1)+\mu-\rho-k}$ at $e+1$. Therefore, so that $M$ is symmetrical, it is necessary to have $\mu-\rho-k=k+1$, i.e., $k=\frac{\mu-\rho-1}{2}$. On the other hand, it is easy to verify that the spline $B=\sum_{j=k+1}^{k+\mu-1} N_{j}$ can be written in the form

$$
B= \begin{cases}\sum_{j=0}^{k-1}\left(N_{k+j+1}+N_{\mu+k-(1+j)}\right)+\sum_{j=\mu-\rho}^{\mu-\varrho-1}\left(N_{j}+N_{\mu-1-(j-\mu+\rho)}\right) & \text { if } \rho=2 \varrho, \\ \sum_{j=0}^{k-1}\left(N_{k+j+1}+N_{\mu+k-(1+j)}\right)+\sum_{j=\mu-\rho}^{(\mu-\rho)+\varrho-1}\left(N_{j}+N_{\mu-1-(j-\mu+\rho)}\right)+N_{\mu-\rho+\varrho} & \text { if } \rho=2 \varrho+1 .\end{cases}
$$

Consequently, $B$ is symmetric with respect to $\frac{e+1}{2}$, and so that $M=a_{k} N_{k}+B+a_{k+\mu} N_{k+\mu}$ is symmetric it is 
necessary to have $a_{k}=a_{k+\mu}$. As $a_{k}+a_{k+\mu}=1$, we deduce that $a_{k}=a_{k+\mu}=\frac{1}{2}$.

We conclude that $M=\frac{1}{2} N_{k}+B+\frac{1}{2} N_{k+\mu}, k=\frac{\mu-\rho-1}{2}$, is the unique spline with minimal support which satisfies the properties i), ii), iii) and iv).

When $k>\mu-\rho($ resp. $k \geq \mu-\rho)$ we can easily show in the case $l=\mu$ (resp. $l=\mu+1)$ that the associated spline $M$ is of support $[0, e+2]$, hence it isn't of minimal support.

Remark 2.3. The condition iii) of Theorem 2.2 is necessary to ensure the uniqueness of the function $M$. Indeed, consider the space $S_{6}^{3}$, then we have $d=6, r=3, \mu=3, e=2, \rho=1$. In this case, $M=N_{1}+N_{2}+N_{3}$ and its support is $[0,3]$. Without the condition $i i i)$, we can find another function supported by $[0,3]$ and satisfying the properties $i), i i), i v)$. More specifically, for $\lambda \in[0,1]$, we consider $M^{\lambda}=(1-\lambda) N_{0}+\lambda N_{1}+N_{2}+\lambda N_{3}+(1-\lambda) N_{4}$. It is clear that for $\lambda \in] 0,1\left[, M^{\lambda}\right.$ is a combination of 5 successive B-splines. But for $\lambda=0, M^{0}$ is combination of three B-splines, which are not successive. Hence, $M^{1}=M$ is the only function that satisfies the property $\left.i i i\right)$.

For the approximation order in the space $S(M)$, we have the following result.

Theorem 2.4. The approximation order in the space $S(M)$ is equal to 1 if $\mu \neq 2$ or $\rho \neq 1$ and equal to 2 if $\mu=2$ and $\rho=1$.

Proof. In order to establish this result, we use the notation $N_{i, d+1}$ instead of $N_{i}$ and $M_{d+1}$ instead of $M$. According to the derivative algorithm of the B-splines, $M_{d+1}$ can be written as

$$
M_{d+1}= \begin{cases}\frac{d}{e-1} N_{0, d} * \chi_{[0,1]} & \text { if } \rho=0, \\ \frac{d}{e} N_{\frac{\mu-\rho}{2}, d} * \chi_{[0,1]} & \text { if } \rho>0 \text { and } \mu-\rho \text { is even, } \\ \frac{d}{2 e}\left(N_{\frac{\mu-\rho-1}{2}, d}+N_{\frac{\mu-\rho-1}{2}+1, d}\right) * \chi_{[0,1]} & \text { if } \rho>0 \text { and } \mu-\rho \text { is odd, }\end{cases}
$$

where $*$ denote the convolution product.

Assume that $\mu \neq 2$ or $\rho \neq 1$. If $\rho=0$, we have $M_{d+1}=\frac{d}{e-1} N_{0, d} * \chi_{[0,1]}$.

Since $\frac{d}{e-1} \sum_{i \in \mathbb{Z}} N_{0, d}(\cdot-i) \neq 1$, we deduce that $\frac{d}{e-1} N_{0, d}$ does not satisfy the Strang-Fix conditions of order 1 . On the other hand, $\chi_{[0,1]}$ satisfies the Strang-Fix conditions of order 1 , then the approximation order in the space $S\left(M_{d+1}\right)$ is equal to 1 . A similar technique can be used when $\rho>0$.

Now, assume that $\mu=2$ and $\rho=1$, then we have

$$
M_{d+1}=M_{d} * \chi_{[0,1]} .
$$

Consequently, the approximation order in the space $S\left(M_{d+1}\right)$ is equal to 2 .

\section{Cardinal $\mathrm{B}_{m}$-SPLines}

\subsection{Definitions and properties}

In this section, we are interested in the construction of new B-splines based on $M$ and having higher approximation order. More specifically, we define these functions by recurrence on $m$ and by convolution with $M$ and the characteristic function of $[0,1]$ in the following way

$$
M_{m}=M_{m-1} * \chi_{[0,1]}, \text { for } m \geq 1 \text {, where } M_{0}=M .
$$

As in the case $\mu=1$, it is easy to verify that these B-splines satisfy the following properties.

Theorem 3.1.

(i) $M_{m}$ is symmetric,

(ii) supp $_{m}=\left[0, m_{0}+m\right]$, where $m_{0}=e$ if $\rho=0$ and $e+1$ if $\rho>0$,

(iii) $M_{m}$ is positive on $] 0, m_{0}+m[$,

(iv) $\sum_{j \in \mathbb{Z}} M_{m}(\cdot-j)=1$. 
Moreover, by using the Strang-Fix conditions and Theorem 2.4, we can easily show the following result.

Theorem 3.2. The approximation order in the space $S\left(M_{m}\right)$ is equal to $m+1$ if $\mu \neq 2$ or $\rho \neq 1$ and equal to $m+2$ if $\mu=2$ and $\rho=1$.

From Theorem 2.2, $m_{0}=e$ if $\rho=0$ and $e+1$ if $\rho>0$, this implies that supp $M_{m}=[0, m+e]$ if $\rho=0$ and supp $M_{m}=[0, m+e+1]$ if $\rho>0$. As in the approximation theory, the basis functions with small supports are appreciated, we consider in our case the B-splines $M_{m}$ with $\rho=0$, i.e., $d+1=\mu e$. Moreover, the choice of $e$ as small as possible leads to B-splines $M_{m}$ with small support [0,m+e]. For examples, for $e=1$, we get $r=-1$ and the corresponding B-splines are discontinuous. For $e=2$, we have $d=2 r+1$ and $\mu=d-r=r+1$, therefore, in order to ensure the existence of B-splines $M_{m}$ with small supports, we must choose $M$ in the Hermite spline space $\mathcal{S}_{2 r+1}^{r}(\rho=0$ and $e=2)$. From Theorem 2.2, we deduce that $M$ is a linear combination of a minimal number of B-splines $\left(N_{i}\right)_{i \in \mathbb{Z}}$ defined in Section 1 with $d=2 r+1$. More specifically, $M=\sum_{j=0}^{r} N_{j}$. As this B-spline $M$ is of class $C^{r}$ and its support is formed by two subintervals, we denote it in the following by $M_{2, r}$.

Proposition 3.3. The explicit expression of $M_{2, r}=\sum_{j=0}^{r} N_{j}$ is given by

$$
M_{2, r}(x)=\left\{\begin{array}{cl}
u_{r}(x) & \text { if } x \in[0,1] \\
u_{r}(2-x) & \text { if } x \in[1,2] \\
0 & \text { elsewhere }
\end{array}\right.
$$

where

$$
u_{r}(x)=\sum_{j=r+1}^{2 r+1}\left(\begin{array}{c}
2 r+1 \\
j
\end{array}\right) x^{j}(1-x)^{2 r+1-j} \quad, \quad x \in[0,1] .
$$

Proof. As $M_{2, r}$ is symmetrical with respect to 1 , it can be written in the form

$$
M_{2, r}(x)=\left\{\begin{array}{cl}
u_{r}(x) & \text { if } x \in[0,1] \\
u_{r}(2-x) & \text { if } x \in[1,2] \\
0 & \text { elsewhere }
\end{array}\right.
$$

where the expression of $u_{r}(x)$ in the Bernstein basis is

$$
u_{r}(x)=\sum_{j=r+1}^{2 r+1} a_{2 r+1}^{r}(j)\left(\begin{array}{c}
2 r+1 \\
j
\end{array}\right) x^{j}(1-x)^{2 r+1-j} \quad, \quad x \in[0,1] .
$$

On the other hand, from (iv) of Theorem 2.2, we have for all $x \in[0,1]$

$$
u_{r}(x)+u_{r}(1-x)=1, \text { which implies that } a_{2 r+1}^{r}(j)=1, \text { for } j=0, \ldots, r \text {. }
$$

Hence, we obtain the expression of $M_{2, r}$.

Now, we define the $\mathrm{B}_{m}$-splines $M_{m, r}, m \geq 2$, as follows.

Definition 3.4. For $m, r \in \mathbb{N}, m \geq 2$, the B-splines $M_{m, r}$, called $\mathrm{B}_{m}$-splines, are defined by for $m=2$,

and for $m \geq 3$,

$$
M_{2, r} \in \mathcal{S}_{2 r+1}^{r}
$$

$$
M_{m, r}(x)=\left(M_{m-1, r} * \chi_{[0,1]}\right)(x) .
$$


In the next, we need the following notations. For all $p, q \in \mathbb{Z}$, we denote by - $I=[p, q]$ an interval of $\mathbb{R}$.

- $\mathcal{P}_{r, m}=\mathcal{S}_{2 r+m-1}^{r+m-2}\left(I,\left\{x_{i}\right\}_{i \in \mathbb{Z}} \cap I\right)$ the space of spline functions of degree at most $2 r+m-1$ with knots of multiplicity $r+1$ at $\left\{x_{i}\right\}_{i \in \mathbb{Z}} \cap I$.

- $\mathcal{V}_{r, m}=\left\{s \in \mathcal{P}_{r, m}: s^{(i)}(j)=0, m-1 \leq i \leq r+m-2\right.$ and $\left.j \in I \cap \mathbb{Z}\right\}$.

Lemma 3.5. a) $\operatorname{dim} \mathcal{P}_{r, m}=(r+1)(q-p)+r+m-1$.

b) $\operatorname{dim} \mathcal{V}_{r, m}=q-p+r-1$.

Proof. a) It follows from the definition of $\mathcal{P}_{r, m}$ and the results, given in [4], concerning the dimension of spline spaces.

b) Let $s \in \mathcal{P}_{r, m}$. For all $j \in\{p, \ldots, q\}$, we consider the linear forms

$$
\varphi_{i, j}(s)=s^{(i)}(j), i=m-1, \ldots, r+m-2 .
$$

They are linearly independent. Indeed, if we assume that $\psi=\sum_{i=m-1}^{r+m-2} \sum_{j=p}^{q} \alpha_{i, j} \varphi_{i, j}=0$, then the application of $\psi$ to the following functions of $\mathcal{P}_{r, m}$

$$
(x-q+1)_{+}^{r+m-1}, \ldots,(x-q+1)_{+}^{2 r+m-2},
$$

leads to the linear system

$$
\sum_{j=0}^{r-1} \frac{(r+i) !}{(r+i-j) !} \alpha_{m-1+j, q}=0, i=0, \ldots, r-1 .
$$

It is easy to check that the determinant of this system does not vanish, therefore $\alpha_{i, q}=0$, for $i=m-1, \ldots, r+$ $m-2$.

In the same way, by applying $\psi$ respectively to the functions

$$
(x-j+1)_{+}^{r+m-1},(x-j+1)_{+}^{r+m}, \ldots,(x-j+1)_{+}^{2 r+m-2}, j=q-1, \ldots, p+1,
$$

we prove that $\alpha_{i, j}=0$.

Finally, in order to show that $\alpha_{i, 0}=0$, it suffices to apply $\psi$ to $x^{m-1}, x^{m}, \ldots, x^{m+r-2}$. Since $\bigcap_{i, j} \operatorname{ker} \varphi_{i, j}=\mathcal{V}_{r, m}$, we deduce that $\operatorname{dim} \mathcal{V}_{r, m}=q-p+m-1$.

Proposition 3.6. The family $\left\{M_{m, r}(\cdot-j), p+1-m \leq j \leq q-1\right\}$ forms a basis of $\mathcal{V}_{r, m}$.

Proof. It can be easily done by induction on $m$.

In the next subsection, we study the cardinal interpolation based in the centred $\mathrm{B}_{m}$-splines.

\subsection{Cardinal interpolation}

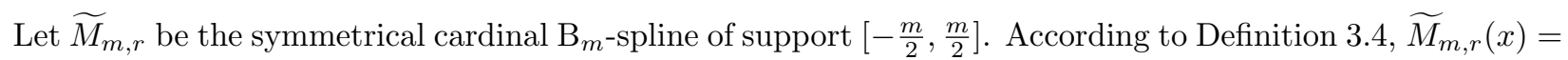
$\left(\widetilde{M}_{2, r} * N_{m-2}\right)(x)$, where $N_{m-2}$ is the symmetrical classical cardinal B-spline of order $m-2$, and its Fourier transform is given by

$$
\widehat{N}_{m-2}(\omega)=\left(\frac{\sin \frac{\omega}{2}}{\frac{\omega}{2}}\right)^{m-2}=\left(\operatorname{sinc} \frac{\omega}{2}\right)^{m-2}
$$


In this section, we are interested in the cardinal interpolation problem in the space $S\left(\widetilde{M}_{m, r}\right)$. According to [14], to ensure the correctness of the interpolation problem with the $\mathrm{B}_{m}$-spline $\widetilde{M}_{m, r}$, i.e., the existence and the uniqueness of the solution of

$$
\sum_{i \in \mathbb{Z}} a_{i}\left(\widetilde{M}_{m, r}\right) \widetilde{M}_{m, r}(j-i)=f(j), \text { for all } j \in \mathbb{Z}
$$

where $f$ is a sufficiently smooth function, it is necessary and sufficient to show that the symbol $\Lambda_{m}^{r}(w)=$ $\sum_{j \in \mathbb{Z}} \widetilde{M}_{m, r}(j) e^{-\imath w j}$ of $\widetilde{M}_{m, r}$ does not vanish. To do this, we need the following lemma.

Lemma 3.7. Let $p_{r}$ be the function defined by

$$
p_{r}(x)=\left\{\begin{array}{cl}
(2 r+1)\left(\begin{array}{c}
2 r \\
r
\end{array}\right)\left(\frac{1}{2}+x\right)^{r}\left(\frac{1}{2}-x\right)^{r} & \text { if } x \in\left[-\frac{1}{2}, \frac{1}{2}\right] \\
0 & \text { otherwise }
\end{array}\right.
$$

Then, for each $\left.\omega \in] 0, \frac{1}{2}\right]$ and $r \geq 1$, we have

(i) $\widehat{p}_{r}(\omega)=\left(\sum_{s=\left[\frac{r+1}{2}\right]}^{r} \alpha_{s}^{r} \frac{1}{\omega^{2 s+1}}\right) \sin \frac{\omega}{2}+\left(\sum_{s=\left[\frac{r}{2}\right]}^{r-1} \beta_{s}^{r} \frac{1}{\omega^{2 s+2}}\right) \cos \frac{\omega}{2}$,

with $\alpha_{s}^{r}=(-1)^{s+r} 2 \frac{(2 r+1) !}{(2 r-2 s) !}\left(\begin{array}{c}2 s \\ r\end{array}\right), \beta_{s}^{r}=(-1)^{s+r} 2 \frac{(2 r+1) !}{(2 r-2 s-1) !}\left(\begin{array}{c}2 s+1 \\ r\end{array}\right)$.

(ii) $\quad \widehat{p}_{r}(2 \pi \omega) \geq \widehat{p}_{r}(\pi) \geq \widehat{p}_{1}(\pi)=\frac{24}{\pi^{3}}$.

(iii) $\quad \widehat{p}_{r}(2 \pi(\omega-1)) \geq \widehat{p}_{r}(2 \pi) \geq \widehat{p}_{1}(2 \pi)=\frac{3}{\pi^{2}}$.

Proof. (ii) We remark that $\widehat{p}_{r}(2 \pi \omega)=2 \int_{0}^{\frac{1}{2}} p_{r}(x) \cos 2 \pi \omega x d x$. Then, as $\left.\left.\omega \in\right] 0, \frac{1}{2}\right]$ we deduce that $\widehat{p}_{r}(2 \pi \omega) \geq$ 0 . Moreover, it is easy to check that the function $\widehat{p}_{r}(2 \pi$.) is decreasing on $\left.] 0, \frac{1}{2}\right]$, so $\widehat{p}_{r}(2 \pi \omega) \geq \widehat{p}_{r}(\pi)$.

On the other hand, by using the expression of $\widehat{p}_{r}$ given in (i) of Lemma 3.7, we obtain

$$
\widehat{p}_{r}(\pi)=\sum_{s=\left[\frac{r+1}{2}\right]}^{r} \alpha_{s}^{r} \frac{1}{\pi^{2 s+1}}
$$

and since $\pi$ is a transcendent number, we have $\widehat{p}_{r}(\pi)>0$.

To show that $\widehat{p}_{r}(\pi) \geq \widehat{p}_{1}(\pi)$ for each $r \geq 1$, it suffices to prove that the sequence $\left(\widehat{p}_{r}(\pi)\right)_{r}$ is increasing. Indeed, if we integrate by parts two times $\widehat{p}_{r}(\omega)$ and we use the fact that $p_{r+2}\left(\frac{1}{2}\right)=p_{r+2}^{\prime}\left(\frac{1}{2}\right)=0$, we obtain

$$
\widehat{p}_{r+2}(\omega)=-2 \int_{0}^{\frac{1}{2}} p_{r+2}^{\prime \prime}(x) \frac{\cos \omega x}{\omega^{2}} d x
$$

So, $p_{r+2}^{\prime \prime}$ can be written as follows

$$
p_{r+2}^{\prime \prime}(x)=(2 r+5)(2 r+4)\left[q_{r+1}(x)-2 p_{r+1}(x)\right], \quad \text { where } \quad q_{r+1}(x)=-2 \frac{r+1}{r+2} p_{r+1}(x)+2 \frac{2 r+3}{r+2} p_{r}(x) .
$$

Therefore

$$
p_{r+2}^{\prime \prime}(x)=4(2 r+5)(2 r+3)\left[p_{r}(x)-p_{r+1}(x)\right], \quad \text { and } \quad \widehat{p}_{r+2}(\omega)=\frac{4(2 r+5)(2 r+3)}{\omega^{2}}\left[\widehat{p}_{r+1}(\omega)-\widehat{p}_{r}(\omega)\right] .
$$

As $\widehat{p}_{r+2}(\pi)>0$, we have for all $r \geq 1 \widehat{p}_{r+1}(\pi)>\widehat{p}_{r}(\pi) \geq \widehat{p}(\pi)=\frac{24}{\pi^{3}}$. 
(ii) One can easily see that $\widehat{p}_{r}(2 \pi(\omega-1)) \geq 0$ for all $\left.\left.\omega \in\right] 0, \frac{1}{2}\right]$, and the function $\widehat{p}_{r}(2 \pi(.-1))$ is increasing on ]0, $\left.\frac{1}{2}\right]$. Therefore, we obtain the following inequality

$$
\widehat{p}_{r}(2 \pi(\omega-1)) \geq \widehat{p}_{r}(-2 \pi)=\widehat{p}_{r}(2 \pi) .
$$

Since $\pi$ is a transcendent number, we have $\widehat{p}_{r}(2 \pi)>0$ for all $r \geq 1$. On the other hand, from equation (5), we get

$$
\widehat{p}_{r+2}(2 \pi)=\frac{(2 r+5)(2 r+3)}{\pi^{2}}\left(\widehat{p}_{r+1}(2 \pi)-\widehat{p}_{r}(2 \pi)\right) .
$$

Then, for all $r \geq 1$, we have

$$
\widehat{p}_{r+1}(2 \pi)>\widehat{p}_{r}(2 \pi) \geq \widehat{p}_{1}(2 \pi)=\frac{3}{\pi^{2}} .
$$

Now, we are in position to prove the main theorem of this section.

Theorem 3.8. The symbol $\Lambda_{m}^{r}$ of the symmetrical cardinal $B_{m}$-spline $\widetilde{M}_{m, r}$ is positive.

Proof. As $\Lambda_{m}^{r}$ is symmetric and $2 \pi$-periodic, it suffices to prove that $\Lambda_{m}^{r}(2 \pi \omega)>0$, for all $\omega \in\left[0, \frac{1}{2}\right]$.

For $m=2$, it is easy to check that $\Lambda_{2}^{r}(2 \pi \omega)=1$.

For $m \geq 3$, we distinguish two cases.

(i) For $\omega=0$, we have $\Lambda_{m}^{r}(0)=\sum_{j \in \mathbb{Z}} \widetilde{M}_{m, r}(j)=1$.

(ii) For $\left.\omega \in] 0, \frac{1}{2}\right]$, we first prove that $\widehat{\widehat{M}}_{m, r}(2 \pi \omega)$ and $\widehat{\widetilde{M}}_{m, r}(2 \pi(\omega-1))$ are positive. Indeed, as

$$
\widehat{\widetilde{M}}_{m, r}(2 \pi \omega+2 \pi j)=\left(\frac{\sin \pi \omega}{\pi}\right)^{m-1} \frac{(-1)^{j(m-1)}}{(\omega+j)^{m-1}} \widehat{p}_{r}(2 \pi \omega+2 \pi j),
$$

we deduce from Lemma 3.7 that $\widehat{\widetilde{M}}_{m, r}(2 \pi \omega)$ and $\widehat{\widetilde{M}}_{m, r}(2 \pi(\omega-1))$ are positive on $\left.] 0, \frac{1}{2}\right]$. Now, we show that

$$
\sum_{j \neq\{0,-1\}}\left|\widehat{\widetilde{M}}_{m, r}(2 \pi \omega+2 \pi j)\right|<\widehat{\widetilde{M}}_{m, r}(2 \pi \omega)+\widehat{\widetilde{M}}_{m, r}(2 \pi(\omega-1)) .
$$

For this, we set

$$
b_{i}(j)=\frac{\left|\widehat{\widetilde{M}}_{m, r}(2 \pi \omega+2 \pi j)\right|}{\widehat{\widetilde{M}}_{m, r}(2 \pi(\omega-i))}, \text { for } i=0,1 \text { and } j \in \mathbb{Z}
$$

and we prove that $\sum_{\substack{j>0 \\ j<-2}} b_{0}(j)<1$ and $b_{1}(-2)<1$. Indeed, from the expression of $\widehat{\widetilde{M}}_{m, r}$, we have

$$
b_{0}(j)=\left|\frac{\omega}{\omega+j}\right|^{m-1} \frac{\left|\widehat{p}_{r}(2 \pi \omega+2 \pi j)\right|}{\widehat{p}_{r}(2 \pi \omega)} .
$$

According to (i) of Lemma 3.7 and the fact that

$$
\left|\widehat{p}_{r}(2 \pi \omega+2 \pi j)\right| \leq 2 \int_{0}^{\frac{1}{2}} p_{r}(x)|\cos (2 \pi \omega+2 \pi j) x| d x \leq 1,
$$


we obtain the following inequality

So, for all $j>0$, we have

$$
b_{0}(j) \leq \frac{1}{\widehat{p}_{1}(\pi)}\left|\frac{\omega}{\omega+j}\right|^{m-1}
$$

$$
b_{0}(j) \leq \frac{1}{\widehat{p}_{1}(\pi)}\left(\frac{\omega}{\omega+j}\right)^{m-1} \leq \frac{1}{\widehat{p}_{1}(\pi)}\left(\frac{1}{2 j+1}\right)^{m-1} .
$$

Since $\left(\frac{1}{\hat{p}_{1}(\pi)} \frac{1}{(2 j+1)^{m-1}}\right)_{m}$ is a decreasing sequence, we deduce that its large value is $\frac{1}{\widehat{p}_{1}(\pi)} \frac{1}{(2 j+1)^{2}}$. Then, we have $b_{0}(j) \leq \frac{1}{\widehat{p}_{1}(\pi)} \frac{1}{(2 j+1)^{2}}$. Therefore

$$
\sum_{j>0} b_{0}(j) \leq \frac{1}{\widehat{p}_{1}(\pi)} \sum_{j=1}^{\infty} \frac{1}{(2 j+1)^{2}}=\frac{1}{\widehat{p}_{1}(\pi)}\left(\frac{\pi^{2}}{8}-1\right) .
$$

In the same way, if $j<-2$, we prove that

$$
b_{0}(j) \leq \frac{1}{\widehat{p}_{1}(\pi)}\left(\frac{-\omega}{\omega+j}\right)^{m-1} \leq \frac{1}{\widehat{p}_{1}(\pi)}\left(\frac{-1}{2 j+1}\right)^{m-1} \leq \frac{1}{\widehat{p}_{1}(\pi)} \frac{1}{(2 j+1)^{2}},
$$

hence

$$
\sum_{j<-2} b_{0}(j) \leq \frac{1}{\widehat{p}_{1}(\pi)} \sum_{j=3}^{\infty} \frac{1}{(2 j-1)^{2}}=\frac{1}{\widehat{p}_{1}(\pi)}\left(\frac{\pi^{2}}{8}-\frac{10}{9}\right) .
$$

Combining the inequalities (6) and (7), we obtain

$$
\sum_{\substack{j>0 \\ j<-2}} b_{0}(j) \leq \frac{\pi^{3}}{24}\left(\frac{\pi^{2}}{4}-\frac{19}{9}\right)=0.46 \ldots<1 .
$$

On the other hand, we have

$$
b_{1}(-2)=\left|\frac{\omega-1}{\omega-2}\right|^{m-1} \frac{\left|\widehat{p}_{r}(2 \pi(\omega-2))\right|}{\widehat{p}_{r}(2 \pi(\omega-1))},
$$

and from (ii) of Lemma 3.7 we get

$$
b_{1}(-2) \leq \frac{1}{\widehat{p}_{1}(2 \pi)}\left(\frac{1-\omega}{2-\omega}\right)^{m-1} \leq \frac{1}{\widehat{p}_{1}(2 \pi)} \frac{1}{2^{m-1}} \leq \frac{\pi^{2}}{12}=0.82 \ldots<1 .
$$

Hence

$$
\sum_{\substack{j>0 \\ j<-2}}\left|\widehat{\widetilde{M}}_{m, r}(2 \pi \omega+2 \pi j)\right|<\widehat{\widetilde{M}}_{m, r}(2 \pi \omega) \text { and }\left|\widehat{\widetilde{M}}_{m, r}(2 \pi(\omega-2))\right|<\widehat{\widetilde{M}}_{m, r}(2 \pi(\omega-1)) .
$$

So, according to the Poisson summation formula, the symbol $\Lambda_{m}^{r}$ can be written in the form $\Lambda_{m}^{r}(\omega)=$ $\sum_{j \in \mathbb{Z}} \widehat{\widehat{M}}_{m, r}(\omega+2 \pi j)$ and therefore $\Lambda_{m}^{r}(2 \pi \omega)>0$. This completes the proof of the theorem.

Remark 3.9. The cardinal fundamental spline $L_{m}^{r}$ and the interpolant operator associated with the $\mathrm{B}_{m}$-spline $\widetilde{M}_{m, r}$ are given respectively by

$$
L_{m}^{r}=\sum_{j \in \mathbb{Z}} c_{j}\left(\widetilde{M}_{m, r}\right) \widetilde{M}_{m, r}(\cdot-j), \quad\left(\mathcal{I}_{m}^{r} f\right)=\sum_{j \in \mathbb{Z}} f(j) L_{m}^{r}(\cdot-j) .
$$


According to the previous sections, the approximation order of $\mathcal{I}_{m}^{r}$ in the space $S\left(\widetilde{M}_{m, r}\right)$ is equal to $m-1$. More specifically, for a sufficiently smooth function $f$ and for $h>0$, there exists a constant $C_{m}^{r}(f)$, independent of $h$, such that

$$
\left\|\mathcal{I}_{m, h}^{r} f-f\right\|_{\infty, J} \leq C_{m}^{r}(f) h^{m-1}
$$

where $\mathcal{I}_{m, h}^{r}=\sigma_{h} \mathcal{I}_{m}^{r} \sigma_{1 / h}$ and $\|\cdot\|_{\infty, J}$ is the uniform norm on the compact $J$ of $\mathbb{R}$.

In order to determine $C_{m}^{r}(f)$, it is necessary to know the expression of $L_{m}^{r}$, or in other words, the coefficients $c_{j}\left(\widetilde{M}_{m, r}\right)$. As it is difficult to compute explicitly these coefficients for all $r$ and $m$, we give only some examples of this computation in the next section.

\section{EXAMPLES}

As examples, we compute the coefficients $\left(c_{j}\left(\widetilde{M}_{m, r}\right)\right)_{j \in \mathbb{Z}}$ of the fundamental splines $L_{m}^{r}$ associated with the cardinal $\mathrm{B}_{m}$-splines $\widetilde{M}_{m, r}$, for all $r \geq 0$ and $m=3,4$. In order to do this, we first prove the following lemma.

Lemma 4.1. Let $r \in \mathbb{N}$. If we denote $\alpha_{r}=\widetilde{M}_{3, r}(-1)=\widetilde{M}_{3, r}(1)$ and $\beta_{r}=\widetilde{M}_{3, r}(0)$, then we have

(i) $\alpha_{r}=\frac{1}{2^{2 r+4}}\left(\begin{array}{c}2 r+2 \\ r+1\end{array}\right)$ and $\beta_{r}=1-2 \alpha_{r}$,

(ii) the sequence $\left(\alpha_{r}\right)_{r}$ is decreasing and the sequence $\left(\beta_{r}\right)_{r}$ is increasing.

Proof. (i) Let $\{a(j), 0 \leq j \leq 2 r+2\}$ (resp. $\{b(j), 0 \leq j \leq 2 r+2\}$ ) be the B-net of the $\mathrm{B}_{3}$-spline $M_{3, r}$ on $[0,1]$ (resp. on $[1,2]$ ). Using the computational algorithm given in [2], we get

$$
a(j)=\left\{\begin{array}{cl}
0 & \text { for } j=0, \ldots, r+1, \\
\frac{j-(r+1)}{2 r+2} & \text { for } j=r+2, \ldots, 2 r+2,
\end{array} \quad \text { and } \quad b(j)=\left\{\begin{array}{cl}
\frac{j+r+1}{2 r+2} & \text { for } j=0, \ldots, r+1 \\
\frac{3 r+3-j}{2 r+2} & \text { for } j=r+2, \ldots, 2 r+2 .
\end{array}\right.\right.
$$

Therefore

$$
\alpha_{r}=\widetilde{M}_{3, r}(-1)=\frac{1}{2^{2 r+4}}\left(\begin{array}{c}
2 r+2 \\
r+1
\end{array}\right), \quad \text { and } \beta_{r}=\widetilde{M}_{3, r}(0)=M_{3, r}\left(\frac{3}{2}\right)=1-\frac{1}{2^{2 r+3}}\left(\begin{array}{c}
2 r+2 \\
r+1
\end{array}\right)=1-2 \alpha_{r} .
$$

(ii) From the expression of $\alpha_{r}$, we have $\frac{\alpha_{r+1}}{\alpha_{r}}=\frac{2 r+3}{2 r+4}<1$, thus $\left(\alpha_{r}\right)$ is decreasing and $\left(\beta_{r}\right)$ is increasing.

\subsection{Computation of $c_{j}\left(\widetilde{M}_{3, r}\right), j \in \mathbb{Z}$ and $r \geq 0$}

The symbol $\widetilde{c}(\omega)$ associated with the sequence $\left(c_{j}\left(\widetilde{M}_{3, r}\right)\right)_{j}$ is given by $\widetilde{c}(\omega)=\frac{1}{\Lambda_{3}^{r}(\omega)}$.

If we put $z=e^{-\imath \omega}$, then we have

$$
\Lambda_{3}^{r}(\omega)=\alpha_{r} z^{-1}+\beta_{r}+\alpha_{r} z, \text { and therefore } \widetilde{c}(\omega)=\frac{\frac{1}{\alpha_{r}} z}{1+\frac{\beta_{r}}{\alpha_{r}} z+z^{2}} .
$$

From (ii) of Lemma 4.1, we obtain $\alpha_{r} \leq \frac{1}{8}$ and $\beta_{r} \geq \frac{3}{4}$. Thus, the equation $1+\frac{\beta_{r}}{\alpha_{r}} z+z^{2}=0$ has two real distinct roots given by

$$
z_{1}=\frac{2 \alpha_{r}-1+\sqrt{1-4 \alpha_{r}}}{2 \alpha_{r}} \text { and } z_{2}=\frac{2 \alpha_{r}-1-\sqrt{1-4 \alpha_{r}}}{2 \alpha_{r}}
$$


So, the symbol $\widetilde{c}(\omega)$ can be written in the form

$$
\widetilde{c}(\omega)=\frac{1}{\sqrt{1-4 \alpha_{r}}}\left(\frac{z_{1}}{z-z_{1}}-\frac{z_{2}}{z-z_{2}}\right) .
$$

Using the entire series expansion of $\widetilde{c}(\omega)$, we obtain

$$
\widetilde{c}(\omega)=\frac{1}{\sqrt{1-4 \alpha_{r}}} \sum_{j=-\infty}^{+\infty}\left(\frac{2 \alpha_{r}-1+\sqrt{1-4 \alpha_{r}}}{2 \alpha_{r}}\right)^{|j|} z^{j} .
$$

Hence, the coefficients $c_{j}\left(\widetilde{M}_{3, r}\right), j \in \mathbb{Z}$, are given by

$$
c_{j}\left(\widetilde{M}_{3, r}\right)=\frac{1}{\sqrt{1-4 \alpha_{r}}}\left(\frac{2 \alpha_{r}-1+\sqrt{1-4 \alpha_{r}}}{2 \alpha_{r}}\right)^{|j|} .
$$

4.2. Computation of $c_{j}\left(\widetilde{M}_{4, r}\right), j \in \mathbb{Z}$ and $r \geq 0$

In this case, we give an explicit formula of the coefficients $c_{j}\left(\widetilde{M}_{4, r}\right)$ of the cardinal fundamental spline $L_{4}^{r}$ associated with the $\mathrm{B}_{4}$-spline $\widetilde{M}_{4, r}$, for a fixed $r \geq 0$.

The symbol $\widetilde{c}(\omega)$ associated with the sequence $\left(c_{j}\left(\widetilde{M}_{4, r}\right)\right)_{j}$ is given by $\widetilde{c}(\omega)=\frac{1}{\Lambda_{4}^{r}(\omega)}$.

If we put $z=e^{-\imath \omega}$, then we have $\Lambda_{4}^{r}(\omega)=\frac{r+2}{4(2 r+3)} z^{-1}+\frac{3 r+4}{2(2 r+3)}+\frac{r+2}{4(2 r+3)} z$, and therefore

$$
\widetilde{c}(\omega)=\frac{\frac{4(2 r+3)}{r+2} z}{1+\frac{2(3 r+4)}{r+2} z+z^{2}}=\sqrt{\frac{2 r+3}{r+1}}\left(\frac{z_{1}}{z-z_{1}}-\frac{z_{2}}{z-z_{2}}\right)
$$

where $z_{1}=\frac{-(3 r+4)+2 \sqrt{(r+1)(2 r+3)}}{r+2}$ and $z_{2}=\frac{-(3 r+4)-2 \sqrt{(r+1)(2 r+3)}}{r+2}$.

Using the entire series expansion of $\widetilde{c}(\omega)$, we obtain

$$
\widetilde{c}(\omega)=\sqrt{\frac{2 r+3}{r+1}} \sum_{j=-\infty}^{+\infty}\left(-\frac{3 r+4}{r+2}+\frac{2 \sqrt{(r+1)(2 r+3)}}{r+2}\right)^{|j|} z^{j} .
$$

Therefore, the expression of $c_{j}\left(\widetilde{M}_{4, r}\right)$ is given by

$$
c_{j}\left(\widetilde{M}_{4, r}\right)=\sqrt{\frac{2 r+3}{r+1}}\left(-\frac{3 r+4}{r+2}+\frac{2 \sqrt{(r+1)(2 r+3)}}{r+2}\right)^{|j|}, \quad j \in \mathbb{Z} .
$$

\section{REMARKS}

1- As we have said before, it is difficult to compute the coefficients $\left(c_{j}\left(\widetilde{M}_{m, r}\right)\right)_{j \in \mathbb{Z}}$ for all $m$, even if $r=0$. Therefore, it is out the question to construct explicitly the cardinal fundamental splines of high regularity associated with the classical cardinal B-splines. But, for the $\mathrm{B}_{m}$-splines $\widetilde{M}_{3, r}$ and $\widetilde{M}_{4, r}, r \geq 0$, we give the explicit values of $c_{j}\left(\widetilde{M}_{3, r}\right)$ and $c_{j}\left(\widetilde{M}_{4, r}\right), j \in \mathbb{Z}$, and moreover, the corresponding cardinal fundamental splines $L_{3}^{r}$ and $L_{4}^{r}$ are respectively of class $\mathcal{C}^{r+1}$ and $\mathcal{C}^{r+2}$, i.e., of high regularity on $\mathbb{R}$. 
2- According to their expressions, the coefficients $\left(c_{j}\left(\widetilde{M}_{m, r}\right)\right)_{j \in \mathbb{Z}}$, of cardinal fundamental splines $L_{m}^{r}, m=3$, 4 , can be written in the form: $c_{j}\left(\widetilde{M}_{m, r}\right)=\mu_{m}^{r}\left(\theta_{m}^{r}\right)^{|j|}, j \in \mathbb{Z}$. Therefore, the sequence $\left(c_{j}\left(\widetilde{M}_{m, r}\right)\right)_{j}$ decays exponentially as $|j| \longrightarrow \infty$. Moreover, it is easy to see that this sequence is decreasing on $r$. Consequently, the coefficients $c_{j}\left(\widetilde{M}_{m, r}\right)$ converge rapidly to zero when $j$ and $r$ are large.

3 - In the two previous examples, the coefficients $\left(c_{j}\left(\widetilde{M}_{m, r}\right)\right)_{j \in \mathbb{Z}}$ of cardinal fundamental splines $L_{m}^{r}, m=3$ and 4 , are computed by using the entire series expansion of $\frac{1}{\Lambda_{m}^{r}(w)}$. This result can be generalized for all $m$. Indeed, if we denote by $\bar{\Lambda}_{m}^{r}(z)=\sum_{j \in \mathbb{Z}} \widetilde{M}_{m, r}(j) z^{j}$, the extension of the symbol $\Lambda_{m}^{r}$ of $\widetilde{M}_{m, r}$ to the set $\mathbb{C}$, then from Theorem 3.8, $\bar{\Lambda}_{m}^{r}$ is positive on $|z|=1$ and therefore $\frac{1}{\bar{\Lambda}_{m}^{r}}$ has a Laurent series expansion in the set $\left\{z \in \mathbb{Z}: 0<\gamma_{1}<|z|<\gamma_{2}\right\}$ which contains $\{z \in \mathbb{C}:|z|=1\}$, where $] \gamma_{1}, \gamma_{2}[$ is a neighborhood of 1 . Since $\frac{1}{\bar{\Lambda}_{m}^{r}(z)}=\sum_{j \in \mathbb{Z}} c_{j}\left(\widetilde{M}_{m, r}\right) z^{j}$, we deduce all the coefficients $c_{j}\left(\widetilde{M}_{m, r}\right)$ of the cardinal fundamental spline $L_{m}^{r}$.

4- We have studied the upper bounds of norms of interpolant operators $\mathcal{I}_{m}^{r}$, for $m=3,4$ and we have obtained the following results:

$$
\left\|\mathcal{I}_{m}^{r}\right\| \leq c_{m}^{r}, \quad \text { where } c_{3}^{r}=\frac{1}{\bar{\Lambda}_{3}^{r}(-1)}=\frac{1}{1-4 \alpha_{r}} \text { and } c_{4}^{r}=\frac{1}{\bar{\Lambda}_{4}^{r}(-1)}=\frac{2 r+3}{r+1} .
$$

In these two cases, we note that the sequence $\left(c_{m}^{r}\right)_{r}$ is decreasing. Moreover, it converges to 1 when $m=3$ and to 2 in the other case.

In general, according to [14], if $\varphi$ is a compactly supported spline function, $\bar{\varphi}$ its symbol on $\mathbb{C}$, and $\mathcal{I}$ its associated cardinal interpolant operator, then we have $\|\mathcal{I}\| \leq \frac{1}{\overline{\varphi(-1)}}$. Therefore, when $\varphi=\widetilde{M}_{m, r}$ we get

$$
\left\|\mathcal{I}_{m}^{r}\right\| \leq \frac{1}{\bar{\Lambda}_{m}^{r}(-1)}, \text { with } \bar{\Lambda}_{m}^{r}(-1)=\sum_{j \in \mathbb{Z}}(-1)^{j} \widetilde{M}_{m, r}(j)=\sum_{j \in \mathbb{Z}} e^{-\imath \pi j} \widetilde{M}_{m, r}(j) .
$$

Using the Poisson summation formula, we obtain

$$
\bar{\Lambda}_{m}^{r}(-1)=\sum_{j \in \mathbb{Z}} \widehat{\widetilde{M}}_{m, r}(\pi+2 j \pi)=\sum_{j \in \mathbb{Z}} \frac{(-1)^{j(m-1)}}{\left(\frac{2 j+1}{2} \pi\right)^{m-1}} \widehat{p}_{r}((2 j+1) \pi)
$$

and from Lemma 3.7, we get

$$
\bar{\Lambda}_{m}^{r}(-1)=(-1)^{r}\left(\frac{2}{\pi}\right)^{2 m}(2 r+1) ! \sum_{k=\left[\frac{r+1}{2}\right]}^{r}(-1)^{k} \frac{1}{\pi^{2 k}} \frac{\left(\begin{array}{c}
2 k \\
r
\end{array}\right)}{(2 r-2 k) !} \sum_{j \in \mathbb{Z}} \frac{(-1)^{j m}}{(2 j+1)^{m+2 k}} .
$$

In the following table, we have verified that for $m=5,6,7,8$ and for $r=0, \ldots, 6$, the sequence $\left(\frac{1}{\bar{\Lambda}_{m}^{r}(-1)}\right)_{r}$ is decreasing. We conjecture that this result is still true for all $m$ and $r$.

\begin{tabular}{|c|c|c|c|c|c|c|c|}
\hline$r$ & 0 & 1 & 2 & 3 & 4 & 5 & 6 \\
\hline$m=5$ & 4.8000 & 3.9344 & 3.6373 & 3.4856 & 3.3931 & 3.3306 & 3.2856 \\
\hline$m=6$ & 7.5000 & 6.1765 & 5.7273 & 5.5000 & 5.3624 & 5.2703 & 5.2041 \\
\hline$m=7$ & 11.8033 & 9.7040 & 8.9890 & 8.6262 & 8.4063 & 8.2579 & 8.0975 \\
\hline$m=8$ & 18.5294 & 15.2419 & 14.1236 & 13.5569 & 13.2136 & 12.9830 & 12.8175 \\
\hline
\end{tabular}

5- The $\mathrm{B}_{m}$-splines studied in this paper have been generalized to the bivariate case. In [9-11], the authors have showed the existence of simple B-splines of class $\mathcal{C}^{k}$ and small supports on three and four directional 
meshes of $\mathbb{R}^{2}$. These simple B-splines are used afterwards for the construction by convolution of composed B-splines which generalize the classical box-splines on $\mathbb{R}^{2}$ and which are useful for solving some interpolation and approximation problems. A particular application of one type of these composed B-splines is given in [1].

\section{This work is supported by PROTARS III D11/18.}

\section{REFERENCES}

[1] E.B. Ameur and D. Sbibih, Local cardinal interpolation by bivariate H-splines on a three-direction mesh, submitted.

[2] E.B. Ameur, Construction d'interpolants splines cardinaux et décomposition en bases d'ondelettes des surfaces fermées sur la sphère, Thèse, Université de Oujda (2002).

[3] D. Barrera-Rosillo, Esquemas Locales de interpolación de Lagrange y Hermite. Su extensión a dos variables, Tesis doctoral, Granada, (1997).

[4] C. de Boor, A practical guide to splines, Springer Verlag, Berlin (1978).

[5] C.K. Chui, An introduction to Wavelets, Academic Press, Inc (1992).

[6] T.N.T. Goodman and S.L. Lee, Wavelets of multiplicity r, Transactions of AMS 342(1) (1994), $307-324$.

[7] T.N.T. Goodman and S.L. Lee, Refinable vectors of spline functions, in Mathematical Methods for Curves and Surfaces II, Morten Daehlen, Tom Lyche, Larry L.Schumaker (eds.), Nashville (1998), 213-220.

[8] T.N.T. Goodman, Refinable spline functions and Hermite interpolation, in Mathematical Methods for Curves and Surfaces, T.Lyche and L.L.Schumaker (eds.), Nashville (2001), 147-161.

[9] A. Mazroui, P. Sablonnière and D. Sbibih, Existence and construction of $H_{1}$-splines of class $C^{k}$ on a three directional mesh, Adv. Comput. Math. 17 (2002), 167-198.

[10] A. Mazroui and D. Sbibih, Existence and construction of $\Delta_{1}$-splines of class $C^{k}$ on a three directional mesh, Numer. Algorithms 26 (2001), 21-48.

[11] O. Nouisser and D. Sbibih, Existence and construction of simple B-splines of class $C^{k}$ on a four directional mesh of the plane, Numer. Algorithms. 27 (2001), 329-358.

[12] P. Sablonnière and D. Sbibih, Spline integral operators exact on polynomials, Approximation Theory and its Applications, vol.10 n.3 (1994) 56-73.

[13] D. Sbibih, Approximation des fonctions d'une ou deux variables par des opérateurs splines integraux, These, Université de Rennes (1987).

[14] I.J. Schœenberg, Cardinal spline interpolation, SIAM Publ., Philadelphia, Pa.(1973).

[15] L.L. Schumaker, Spline functions: Basic theory, Wiley, New York, (1981).

[16] G. Strang and G. Fix, A Fourier analysis of the finite element variational method, in constructive aspects of functional analysis, G.Geymonat et al. (eds), CIME II Ciclo,(1973) 793-840. 